\title{
Análisis de la percepción de los valores de los estudiantes de la Universidad de Ferrara
}

\author{
Analysis of the perception of the values of the students of the \\ University of Ferrara
}

\author{
(iD) Andrea Cívico Ariza \\ Área de Educación, Universidad Internacional de Valencia, España \\ acivico@universidadviu.com \\ Giorgio Poletti \\ Departamento Estudios Humanísticos, Universidad de Ferrara, Italia \\ giorgio.poletti@unife.it \\ Anita Gramigna \\ Departamento Estudios Humanísticos, Universidad de Ferrara, Italia \\ grt@unife.it \\ (iD) Erika González García \\ Departamento de Pedagogía, Universidad de Granada, España \\ erikag@ugr.es
}

\section{RESUMEN}

Los profesionales de la educación a través de las funciones que desempeñan en su día a día, ejercen una gran influencia en la construcción axiológica de los educandos. Por este motivo, el objetivo de este estudio consiste en analizar la percepción de los valores que presenta el alumnado del Grado en Pedagogía de la Universidad de Ferrara (Italia) como futuros trabajadores del ámbito educativo. Para ello se ha utilizado una metodología cuantitativa con carácter descriptivo. La muestra está conformada por 109 estudiantes del primer curso del grado y la recogida de la información se ha realizado a través del cuestionario estandarizado denominado "Test de Valores Adaptado". Los resultados reflejan un mayor agrado por parte de los estudiantes de las categorías de valores moral, afectivo e individual, mientras que las que han obtenido un menor agrado han sido las relacionadas con los valores individuales, de participación política y religiosos. Estos resultados enclavados dentro de la sociedad postmodernista actual, reflejan la necesidad de potenciar la educación axiológica en todos los niveles educativos.

PALABRAS CLAVE valores, educación, universidad.

\section{ABSTRACT}

Education professionals, through the functions they perform in their day-to-day life, exert a great influence on the axiological construction of students. For this reason, the objective of this study is to analyze the perception of the values presented by the students of the Degree in Pedagogy at the University of Ferrara (Italy) as future workers in the educational field. For 
this, a descriptive quantitative methodology has been used. The sample is made up of 109 students from the first year of the degree and the information was collected through the standardized questionnaire called "Adapted Values Test". The results reflect a greater liking on the part of the students of the categories of moral, affective and individual values, while those that have obtained a lesser liking have been those related to individual, political and religious participation values. These results embedded within the current postmodern society, reflect the need to promote axiological education at all educational levels.

KEYWORDS values, education, colleges.

\section{INTRODUCCIÓN}

Los valores permiten enlazar a las personas con la sociedad en la que se encuentran inmersas (Bonilla et al., 2012; Schwartz y Boehnke, 2004). Por ello, tal como indica Álvarez (2005), es imposible percibir la vida de los seres humanos sin los valores, al constituir elementos esenciales de la personalidad de cada individuo. En este sentido, los valores permiten a las personas entender el mundo que les rodea y responsabilizarse de sus acciones, dando mayor relevancia a aquello que hace su vida más fácil y positiva, y rechazando las decisiones que puedan presentar una repercusión negativa tanto para ellos mismos como para sus seres queridos (Gervilla, 2000a). Dicha elección de valores vendrá determinada por la escala de valores propia de cada persona. Esta se construye mediante un proceso de enseñanza-aprendizaje en el que el individuo adquiere aquellos valores que le permiten evolucionar y vivir de forma agradable. En este sentido, Martínez et al. (2002) exponen que todo lo que se relaciona con la persona y la justificación de su existencia "debe ser objeto y objetivo de enseñanza y aprendizaje" (p.24). Este aprendizaje cobra especial interés en la adolescencia y la juventud, ya que es cuando se inicia la construcción identitaria de la persona con las correspondientes elecciones axiológicas que esto conlleva. En este proceso debemos resaltar cuatro agentes coeducativos esenciales: familia, escuela, grupo de pares y medios de comunicación (Flammer y Alsaker, 2005). Estos son los encargados de transmitir los ideales y la cultura, pues generan escenarios y contextos en los que los individuos interiorizan y actúan a partir de sus sistemas de valores (Bronfenbrenner, 2009).

Para nuestro trabajo, nos centraremos en la institución escolar como primer sistema formal en el que la persona inicia su desarrollo axiológico. En este sentido, Goicoechea (2010) recoge en su investigación la necesidad de que los valores sean abordados desde el ámbito educativo. Igualmente, Gramigna y Contreras (2010) muestran la necesidad de implementar un aprendizaje ético que promueva en la juventud la capacidad de diferenciar lo que es incorrecto y lo que es correcto para que la persona actúe en su beneficio, siempre que este esté subordinado a la moral comunitaria (Esteban, 2018). Y es que, en la actualidad, nos encontramos en una sociedad postmodernista en la que prima la visión individualista de los valores, dónde el bienestar personal se sitúa por encima del social y las personas toman sus decisiones en función de sus deseos sin tener en consideración el bien comunitario (Bauman, 2007; Gil, 2001; Esteban, 2018). Por esta cuestión, "educar en valores y aprender a valorar" (Rodríguez-Sabiote et al., 2019, p.111), se convierte en un reto esencial para la pedagogía. La educación en valores busca promover el desarrollo de competencias que permitan a las personas emitir juicios morales teniendo en cuenta aspectos como las posibilidades de comunicación, la experiencia en discernir los valores, la responsabilidad y la oportunidad de interacción (Lüdecke-Plümer,2007). En este mismo sentido, Arango et al. (2014) recogen que la enseñanza de valores desde el ámbito educativo se presenta como un estímulo para que: el alumnado entienda, reflexione 
y aprenda sobre las experiencias vividas; muestre interés por las personas que los rodean y desarrollen sus propios código éticos; comprendan la necesidad de respetarse a ellos mismos y a los valores comunes de la sociedad, como pueden ser la honradez, la justicia y la veracidad; y sean capaces de justificar y responsabilizarse de sus decisiones y sus acciones.

No podemos olvidar que la moral y los valores acompañan a las personas en toda su existencia. Además, los valores caminan de la mano con los principios morales. Como tal, se hace necesario enseñar que las decisiones axiológicas deben atender a las competencias de orden moral y potenciar los juicios morales que fomenten el desarrollo axiológico del alumno. Prestando, en consecuencia, especial atención a este contenido a lo largo de toda la formación educativa de los jóvenes (Rodríguez-Sabiote et al., 2019).

Dentro de la institución escolar, los docentes cuentan con un papel primordial en la transmisión de valores, ya que con la elaboración del material y las explicaciones diarias están transmitiendo sus propias escalas de valores al alumnado sin tener conciencia de ello (Álvarez 2007). La intención del docente en su labor educadora, siguiendo a Prieto (2008), es que el alumnado desarrolle su propio punto de vista de la realidad y pensamiento crítico. Para conseguir esto, es indispensable que el educador sea consciente de esta realidad y, en consecuencia, adquiera el compromiso de añadir a sus tareas diarias la intención de transmitir los conocimientos, evitando las verdades absolutas en su discurso en la medida de lo posible.

Según Salcedo (2012), el lugar más idóneo para que los futuros profesionales inicien la construcción de su identidad profesional es en el ámbito universitario. Es aquí donde el futuro educador debe preguntarse quién soy, qué entiendo por educación y qué profesional del ámbito formativo quiero llegar a ser. Además, este espacio constituye el escenario en el que el alumnado es preparado para ejercer "como agentes transmisores de valores y generadores de cambio" (Álvarez et al., 2007, p.27). Por lo tanto, es en la universidad donde los futuros docentes toman conciencia de su labor y su responsabilidad, siendo en este espacio donde deben entender que su propia visión de la realidad la transmitirán a los discentes. Siguiendo a Esteve (2006), para un desarrollo axiológico adecuado de los estudiantes, el docente debe concebirse como la figura que acompaña y apoya al alumnado durante su proceso de interiorización de los valores.

Partiendo de la realidad presentada, en esta investigación nos centramos en conocer los valores que manifiesta el alumnado universitario que, posteriormente, desarrollarán su labor profesional en el ámbito educativo. Esta es una temática poco abordada desde el ámbito social, político y educativo. No obstante, para poder mejorar la labor docente en la transmisión de valores hacia los educandos se hace necesario conocer qué valores imperan en los futuros profesionales de la educación.

\section{MATERIAL Y MÉTODO}

\subsection{Enfoque metodológico}

La metodología empleada en el estudio es de tipo cuantitativa con carácter descriptivo. Esta metodología, siguiendo a Poletti (2019), presta atención a los eventos y fenómenos medibles que suceden en las personas y en su entorno. En este caso, mediante la recogida de información a través de la aplicación de un cuestionario estandarizado a la muestra, podremos cuantificar y tratar de forma estadística las percepciones de agrado respecto a diferentes categorías axiológicas y los valores que las conforman. Estamos ante un estudio por un lado exploratorio, al no encontrar otras investigaciones que conjuguen esta temática y pobla- 
ción; y por otro lado transversal, al obtenerse los datos de un determinado grupo de sujetos en un solo momento temporal (Cubo et al., 2011; Cohen et al., 2011; Colomo et al., 2020a; Río et al., 2010; Van et al., 2012).

\subsection{Muestra}

La muestra objeto de estudio está conformada por los estudiantes del primer curso del Grado de Pedagogía de la Universidad de Ferrara (Italia). Se trata de una muestra seleccionada de forma intencionada (no probabilística), al tener acceso los investigadores a la misma como docentes de la titulación. El número de estudiantes es de 109 ( $n=109$ ), siendo el 95,4\% mujeres (104) y el 4,6\% hombres (5). Con una edad media de 21,7 años, su rama de conocimiento para el acceso a los estudios universitarios se divide entre técnico/ administrativo (38,5\%), clásico $(38,5 \%)$ y científico $(23 \%)$.

\subsection{Procedimiento}

Para la obtención de los datos se informó a la muestra de las características del estudio durante el segundo cuatrimestre del curso escolar. A través del correo se facilitó el enlace del cuestionario (realizado en Google Forms), estando vigente la recolección de resultados durante tres semanas, siendo la participación voluntaria y anónima.

\subsection{Instrumento}

En este estudio se ha empleado como instrumento para obtener la información el "Test de Reacción Valorativa” de García Hoz (1976), el cuál fue adaptado por Casares (1995), y actualizado recientemente bajo la denominación “Test de Valores Adaptados” (Gervilla y otros, 2018). El cuestionario actual, está fundamentado en el Modelo Axiológico de Educación Integral (MAEI) del profesor Enrique Gervilla (2000a, 2000b, 2004). Este modelo parte de una concepción global del ser humano, orientando la educación a procesos formativos holísticos y humanizadores que abordan todas las esferas y componentes de su realidad para favorecer así un equilibrio y evolución integral del sujeto. Se compone de tres dimensiones fundamentales: el ser humano como animal de inteligencia emocional; el hombre como ser singular y libre en sus decisiones; y la apertura o naturaleza relacional de los humanos.

El test incluye diez tipos de categorías de valores en relación con estas dimensiones: corporales, intelectuales, afectivos, individuales, morales, estéticos, sociales, ecológicos, instrumentales y religiosos. A estas, se le añade la participación política como ámbito sobre el que profundizar y que no estaba contemplado en el MAEI, quedando finalmente el test conformado por 11 categorías. Cada categoría de valores expresada en el cuestionario está comprendida por 25 términos o palabras. A cada sujeto se le solicita que manifieste su grado de conformidad para cada palabra, permitiendo así medir la intensidad positiva y negativa del valor respecto a las percepciones de los encuestados. La cuantía de cada valor se obtiene a través de la sumatoria de cada alternativa de respuesta. Calificándose las respuestas de las siguientes formas: muy agradable (MA), agradable (A), indiferente (I), desagradable (D) o muy desagradable (MD). El valor de cada una de las respuestas oscila entre -2 y +2 puntos, pudiéndose establecer por lo tanto dos rangos en función de la puntuación. Si nos centramos en valorar una categoría en concreto, el rango de la puntuación oscilará entre -50 y +50 puntos. Si embargo, si ponemos el foco en la valoración de un término en concreto, la puntuación fluctuará 
entre -2 y +2 , coincidiendo este rango con el establecido en la escala tipo Likert para la valoración del test.

En cuanto a los criterios de calidad del instrumento utilizado en la recogida de información, utilizaremos los parámetros de validez y fiabilidad. El criterio de validez se garantiza mediante la utilización del mismo en numerosas investigaciones anteriores (Álvarez, 2007; Álvarez y Rodríguez, 2008; Cámara, 2003; Casado y Sánchez-Gey, 1999; Cívico et al., 2019; Rodríguez et al., 2008). La validez criterial se ha obtenido a través de una correlación entre el total de la prueba y cada uno de los 275 ítems que la componen, obteniéndose un predominio de correlaciones estadísticamente significativas, alcanzándose por lo tanto un nivel de confianza del 95\%.

Para medir la fiabilidad del instrumento, se ha empleado el coeficiente alfa de Cronbach. El resultado obtenido ha sido de 0.96 , reflejando este dato la alta consistencia interna de los ítems seleccionados y en consecuencia la alta fiabilidad del cuestionario.

El procesamiento y análisis de los datos obtenidos en la investigación, se ha llevado a cabo mediante la utilización del Paquete Estadístico para Ciencias Sociales (SPSS), en su versión 25 y el programa Microsoft Excel 2019, siguiendo los criterios de puntuación explicados anteriormente.

\section{RESULTADOS Y DISCUSIÓN}

En este apartado se llevará acabo la presentación de los resultados y el análisis y discusión de los mismos. En la siguiente tabla se muestran los estadísticos descriptivos más relevantes de nuestra muestra.

TABLA 1. Estadísticos descriptivos

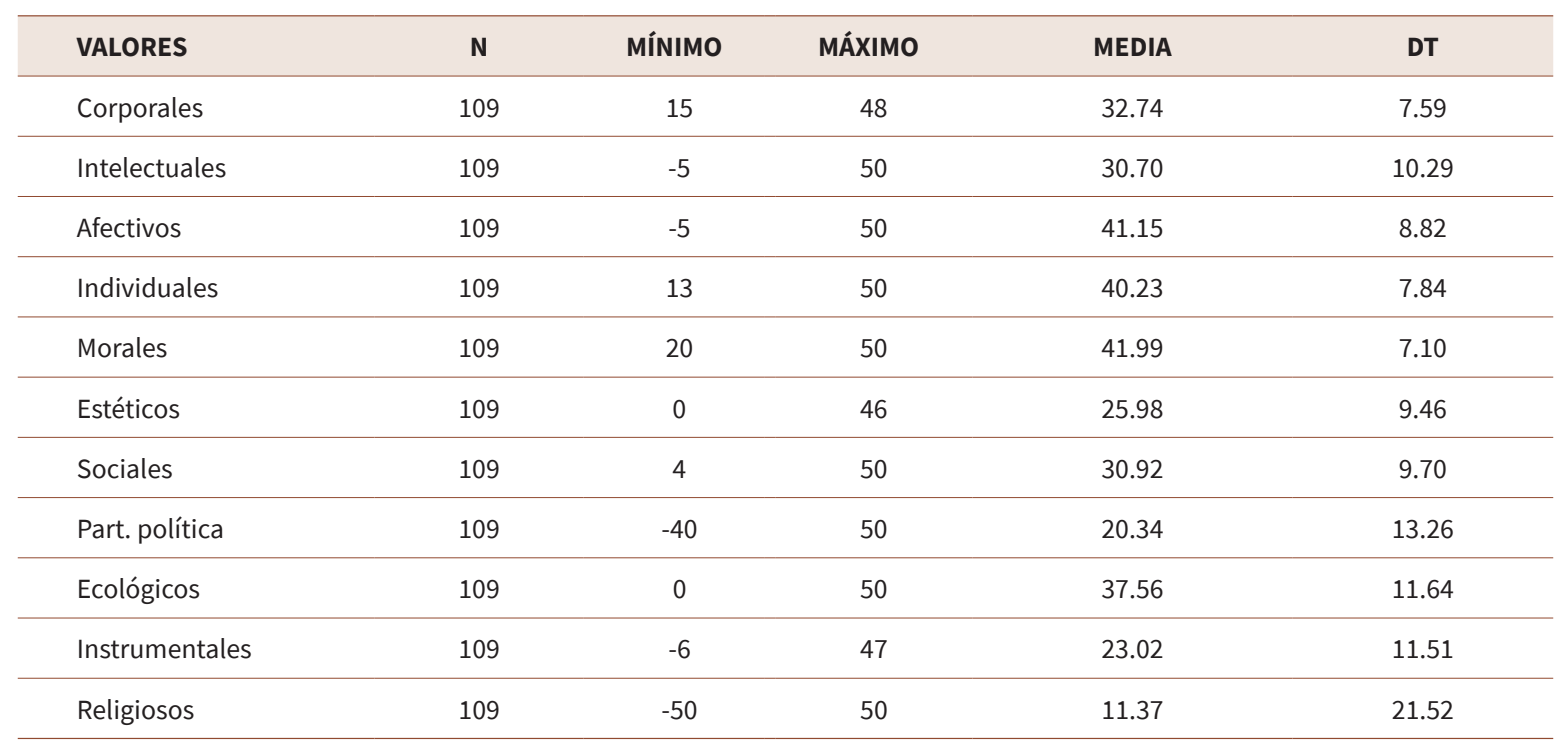

Recordando que la mayor puntuación de una categoría es 50 y la menor -50, es reseñable comentar que en la religiosa se ha alcanzado el mínimo valor posible (-50 puntos), significando que algún estudiante ha considerado muy desagradable todos los términos que componían dicha categoría. De igual forma, son varias las categorías que han alcanzado la máxima puntuación posible (50 puntos), vinculada al máximo nivel de agrado hacia todos los valores que la componían, en este caso, a las categorías intelectual, afectiva, individual, moral, social, participación política, ecológica y religiosa. 
Si nos centramos en las puntuaciones media en función de las elecciones del alumnado, la puntuación más alta la recibe la categoría moral (41.99), seguida de la afectiva (41.15) y la individual (40.23). En este sentido, los estudiantes muestran un mayor nivel de agrado por aquellos valores que promueven una convivencia pacífica y enriquecedora, siempre asegurando tanto el bienestar personal y afectivo de cada persona como su desarrollo identitario. Las preferencias de los jóvenes por estas categorías coinciden con las investigaciones de Álvarez y Rodríguez (2008), Álvarez et al., (2007) y Casado y Sánchez (1999). Este hecho no es extraño, ya que, en referencia a los valores morales, González-Anleo (2017), resalta que un 92.5\% de los jóvenes considera importante el llevar una vida moral digna. Lo mismo ocurre con los valores afectivos e individuales, al concebirse por los jóvenes como un pilar esencial en su construcción identitaria (Elzo, 1999; Cruz y Santiago,1999).

En el nivel intermedio de agrado y desagrado, coincidiendo con el trabajo de Rodríguez-Sabiote et al. (2019), encontramos la categoría ecológica (37.56), corporal (32.74), social (30.92), intelectual (30.70) y estética (25.98). En ellos, podemos resaltar la importancia que tiene para los jóvenes el papel de la naturaleza y, concretamente, la concienciación sobre el cuidado del planeta que habitamos (Herrera-Mendoza et al., 2016). A continuación, el alumnado muestra agrado por valores relacionados con la atención a su físico y su realidad social, siendo prácticamente igual de importante esta última que su desarrollo cognitivo. Los buenos resultados obtenidos por los valores corporales podemos relacionarlos con la importancia que los jóvenes atribuyen al cuidado de su salud (Nava y Ureña, 2017). A cierta distancia de las categorías anteriores se sitúan los valores estéticos, coincidiendo con el estudio de Álvarez (2004), pudiendo atribuirse a su vinculación con el ocio de carácter cultural, el cual es una forma menos habitual de emplear los jóvenes el tiempo libre en la realidad actual.

En las últimas posiciones encontramos a la categoría instrumental (23.02), la de participación política (20.04) y la religiosa (11.37), siendo los valores por los que la población objeto de estudio muestra menor nivel de agrado.

Es de destacar que en una sociedad líquida (Bauman, 2007) y posmodernista, donde prima el individualismo y el ser humano se define más por lo que posee que por lo que es o hace (Lipovetsky, 1990), la categoría instrumental (con valores que son medios para alcanzar otros fines y están vinculados al materialismo y al consumismo), obtenga una valoración tan baja. En este sentido, esta situación rehúsa la propuesta de Rawls (1991), quien liga el desarrollo integral de la especie humana con un consumismo exacerbado.

La posición de la categoría de participación política, responde al desinterés generalizado de la juventud hacia dicho ámbito. En este sentido, una juventud en la que prima lo inmediato, tiene escasa paciencia (Andrés y Fernández, 2016) y prioriza el beneficio personal sobre el social, entiende que no le es rentable el esfuerzo y tiempo que les conllevaría lograr cambios en su realidad desde la dimensión política, siendo este uno de los motivos de la indiferencia ante el ámbito político como reflejan diferentes estudios (GonzálezAnleo 2017; López-Ruiz, 2017).

Ocupando el último lugar encontramos la categoría religiosa, una situación reiterativa en diferentes estudios (Quijano y Lorenzo, 2011; Álvarez, 2002; Álvarez, 2007). Esta percepción negativa de la dimensión religiosa en los jóvenes puede deberse, según Gervilla (1993), a la concepción postmodernista de religiosidad y a la secularización de la sociedad en la que habitamos.

Respecto a las desviaciones típicas, entendiendo estas como "un indicador de cuán homogénea contra heterogénea es una distribución” (Álvarez y Rodríguez, 2008, p.11), podemos observar que la categoría que 
ofrece menor dispersión es la moral (7.09), seguida de la corporal (7.59), individual (7.84) y afectiva (8.82). Es destacable, por otro lado, que la categoría religiosa tiene el mayor grado de variabilidad en las respuestas (21.52), junto con la de participación política (13.26) y la ecológica (11.64).

Una vez analizado los estadísticos descriptivos, consideramos importante profundizar sobre qué valores concretos obtienen las mayores y menores puntuaciones en cada categoría por parte de los estudiantes, de manera que podremos conocer mejor tanto los valores preferentes como los menos apreciados de cada categoría por la población objeto de estudio. A continuación, se presenta una tabla con toda la información.

TABLA 2. Vocablos mejor y peor percibidos por dimensión axiológica

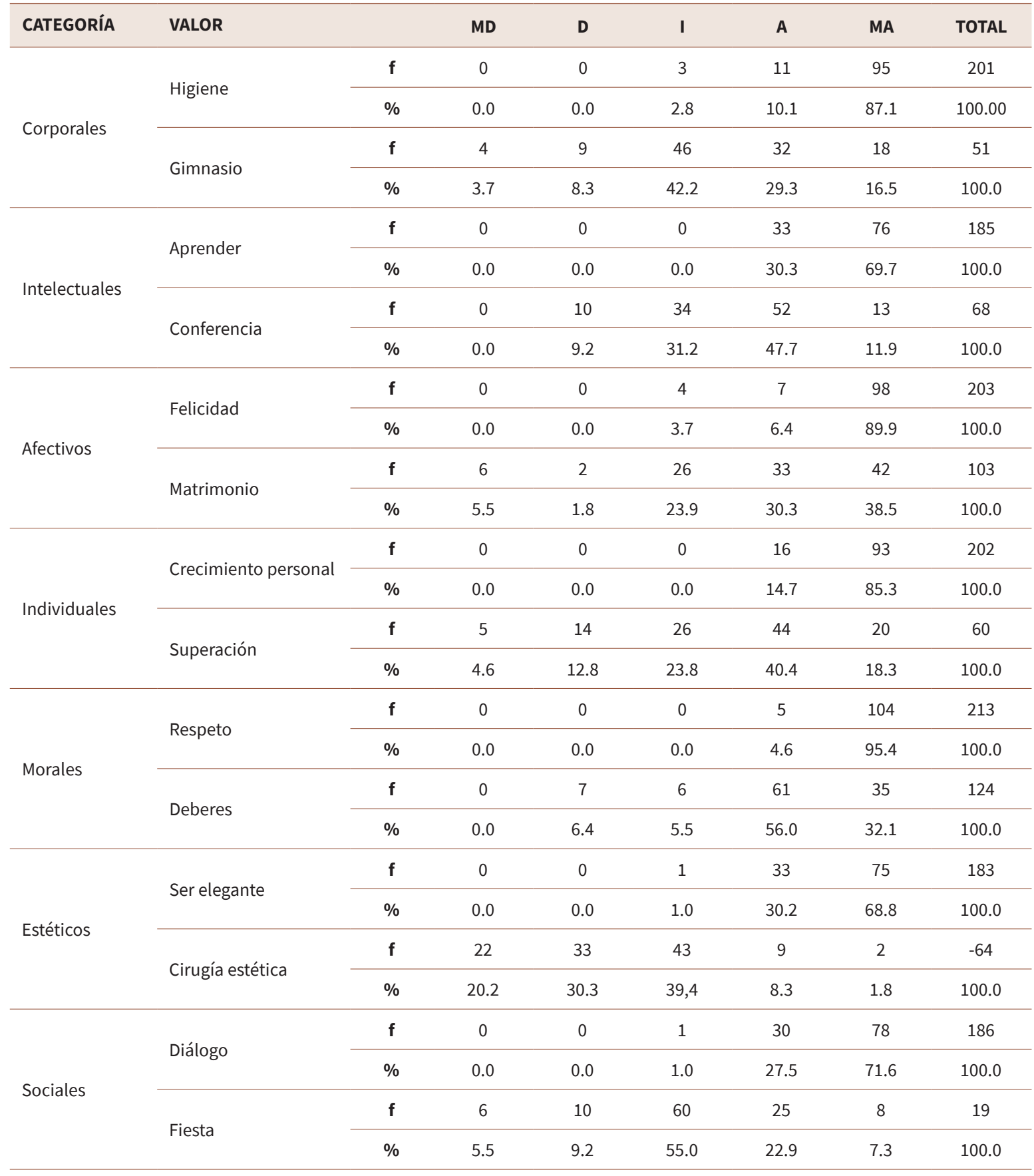




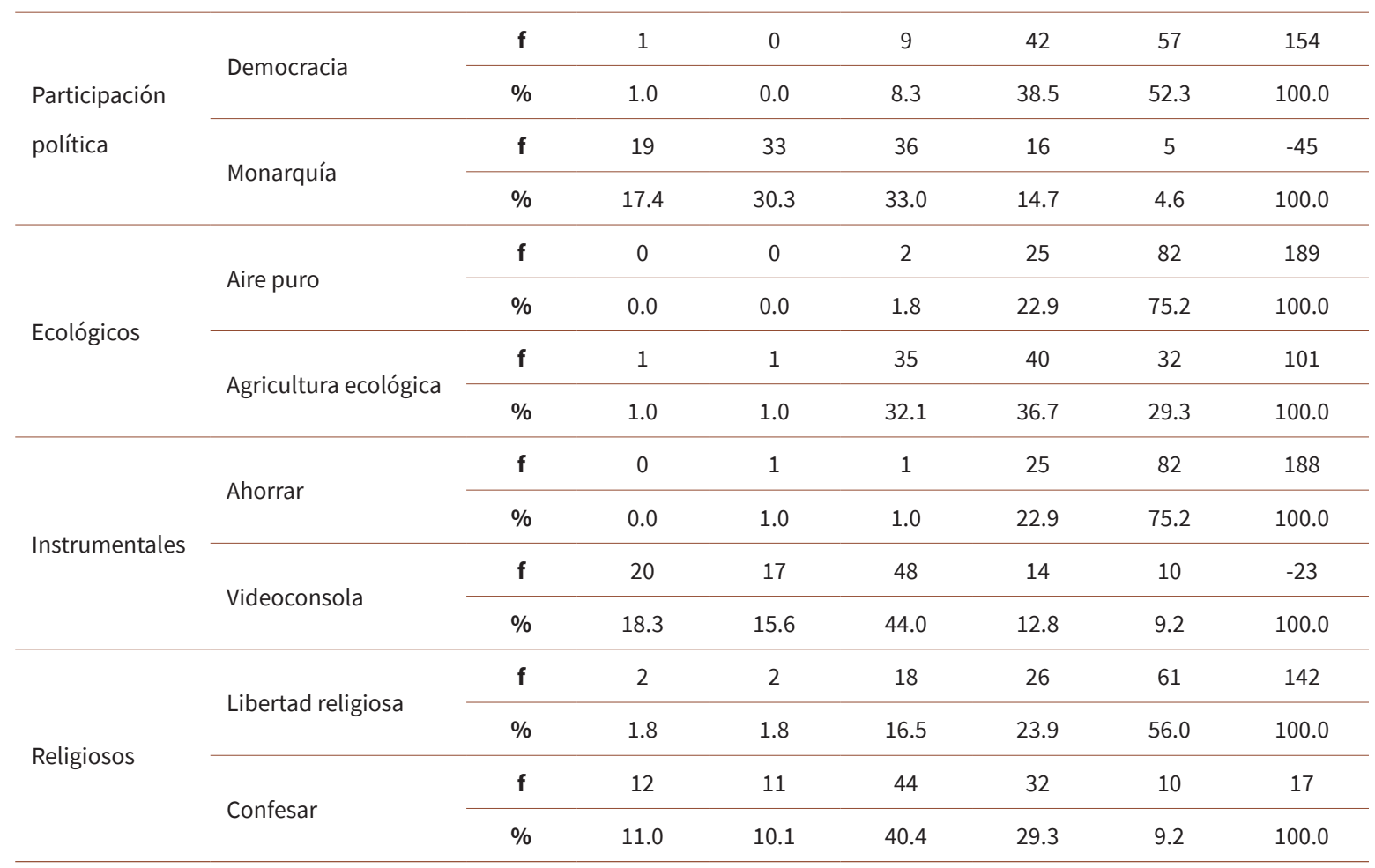

FUENTE: elaboración propia.

En este análisis, es importante recordar que las puntuaciones para recoger el nivel de agrado se sitúan entre el 2 y el -2, tal como se explica en el apartado de descripción del instrumento. En este sentido, dado que la muestra de este estudio está compuesta por 109 estudiantes, las puntuaciones se encuentran entre los 218 y los -218 puntos.

Dentro de la categoría corporal, el valor más puntuado es la higiene, relacionándolo con la importancia que para los jóvenes presenta la imagen y la apariencia física como elemento de aceptación dentro del grupo social de pertenencia (López-Ruiz, 2017). Por el contrario, el valor considerado más desagradable en esta categoría es el gimnasio. Este hecho contradice estudios en los que la mayoría de la juventud declara realizar deporte al conocer los beneficios que este aporta (Codina y Pestana, 2008; Fradejas y Espada, 2018; Gutiérrez et al., 2017). No obstante, podemos atribuir este resultado a la preferencia de los mismos por realizar actividad física en otros entornos en lugar de en el gimnasio.

Para hablar del valor considerado más agradable de la categoría intelectual, es interesante que recordemos que la muestra de este estudio está conformada por alumnos del Grado de Pedagogía y, por lo tanto, su conocimiento y comprensión sobre el proceso de enseñanza-aprendizaje es significativa. Debido a ello, el valor más puntuado en esta categoría es aprender, ya que conciben esta acción como un elemento esencial para crecer en su proceso de construcción identitaria y profesional. No obstante, su concepción de aprendizaje se relaciona más con un proceso dinámico que con uno estático y tradicional, ya que el valor peor considerado es la conferencia, entendiendo esta como una enseñanza unidireccional en la que el proceso de transmisión de información puede resultar poco motivador y sin participación.

La felicidad es el valor mejor puntuado en la categoría afectiva. Este resultado coincide con el estudio de Angelucci et al., (2009), en el que su muestra, también compuesta por estudiantes universitarios, sitúa este valor entre los más preferentes, ya que se relaciona con el desarrollo pleno de la persona y su correcta 
conformación identitaria. En contraposición, el valor considerado como menos agradable en esta categoría es el matrimonio, coincidiendo con la investigación de Álvarez y Rodríguez (2008). Ello puede relacionarse con el hecho de que, en la sociedad actual, este tipo de unión se considera como un trámite cuyos beneficios son posibles de alcanzar sin necesidad de llevarlo a cabo (Hermoza, 2016).

En la categoría individual, el crecimiento personal es el valor mejor considerado, reflejando así la importancia que otorgan a su desarrollo. Sin embargo, existe la contradicción de que el menos puntuado es la superación. En este sentido, la causa puede situarse en relacionar este concepto con un constante desafío interno en diferentes ámbitos, cuya exigencia y la posible no consecución puede ser un factor que les provoque rechazo, desencanto, malestar o una insatisfacción vital.

Respecto a las categorías moral y social, los valores con mayor puntuación son el respeto y el diálogo, respectivamente. Estos constituyen los cimientos de la convivencia pacífica que todos los miembros de la sociedad desean (Colomo et al., 2018), siendo a la vez elemento esenciales de la moral comunitaria (Esteban, 2018) cuyo fin es enriquecer y facilitar una convivencia pacífica, tolerante y respetuosa entre los seres humanos. En cuanto a los valores considerados como menos agradables de ambas categorías, encontramos los deberes y la fiesta. El rechazo a los deberes está en concordancia con una sociedad individualista como es la postmodernista actual, en la cual la asunción de obligaciones se intenta evitar.

Dentro de la categoría estética, se encuentra el valor que presenta mayor desagrado entre todos los analizados, siendo la cirugía estética con una puntuación negativa de -64 puntos. Podemos entender este dato como un rechazo por parte de los jóvenes a la belleza artificial y a todo aquello que se aleje de la naturalidad del propio cuerpo. No obstante, estos resultados contradicen la investigación de Maldonado et al. (2016), que resalta la creciente importancia que, en la adolescencia y la juventud, tiene la cirugía estética como solución a la preocupación por su aspecto físico y mejora de su autoestima. Por su parte, el valor considerado más agradable en esta categoría es ser elegante, reflejándose la importancia de la apariencia exterior. Esta elección presenta relación con el valor destacado también como muy positivo en la categoría corporal, la higiene, pues ambos valores reflejan preocupación, cuidado y compromiso con la apariencia que la persona muestra a la sociedad. En este sentido, se refuta la interpretación realizada sobre la cirugía estética, ya que a la población objeto de estudio si le preocupa su imagen exterior, pero rechaza la artificialidad que las operaciones generan en dicha imagen.

En los resultados de la categoría de participación política, se transmite la importancia que para los jóvenes italianos presenta la soberanía del pueblo y el derecho a elegir libremente a sus gobernantes, siendo el valor más agradable la democracia, mientras que el más desagradable es la monarquía como forma de gobierno no elegida por el pueblo. Estos resultados son muy significativos, pues pese a que la categoría de participación política se sitúe entre las peores valoradas, los jóvenes son conscientes de lo que desean y a lo que se oponen. Este mismo hecho aparece en la elección de valores de la categoría religiosa. Los jóvenes, a pesar de situarla en último lugar, destacan como el valor más deseable la libertad religiosa, es decir, la posibilidad de ser ellos los que elijan en que creer. Mientras que el peor valorado es confesar, rechazando el que una persona ajena a ellos sea quién decida si han obrado bien o mal y les imponga una penitencia a cumplir. No obstante, es importante que, en los resultados de esta categoría, también se tenga presente el concepto de religión a la carta (Lipovetsky, 2016), el cual conlleva la interpretación y configuración personal de las creencias en función de las expectativas de la persona, siendo esta una situación recurrente en nuestra realidad (Cívico et al., 2020). 
En la categoría ecológica, el valor con mayor puntuación es el aire puro mientras que el menor puntuado es la agricultura ecológica. Es necesario subrayar que las puntuaciones de ambas son elevadas, lo que permite refutar nuevamente la importancia que los jóvenes atribuyen a la naturaleza mediante el cuidado del medio ambiente. Por tanto, entendemos que esta diferencia de puntuación puede deberse al desconocimiento de la población juvenil sobre los fundamentos del desarrollo agrícola sostenible.

Por último, comentar la importancia que dentro de la categoría instrumental dan los jóvenes al ahorro, siendo considerado el valor más agradable. Si ponemos el foco en el valor peor considerado, encontramos a la videoconsola, siendo uno de los valores con peor puntuación a nivel global en el test. Este resultado es contrario a las investigaciones de Sánchez et al. (2010), quién resaltaba que la mayoría de los jóvenes basan su ocio en jugar a videojuegos.

\section{CONCLUSIONES}

Considerando que la construcción de la escala de valores de las personas se inicia en la adolescencia y que durante esta etapa las personas permanecen gran parte de su tiempo diario en el sistema educativo, dentro de este contexto, cobra especial relevancia el papel del docente por su influencia en el desarrollo axiológico del alumnado (Álvarez, 2007; Colomo et al., 2020b; Colomo y Aguilar, 2019). Como hemos comentado anteriormente, a través de las explicaciones y el material utilizado, se produce un proceso de transmisión de valores que incide directamente en la conformación de la escala de valores del educando.

Es por ello que, ante esta situación, se haga preciso conocer los valores imperantes en los futuros profesionales del ámbito educativo, como elementos influyentes en la evolución axiológica de los individuos. En este sentido, la categoría de valores que ha obtenido la mayor puntuación ha sido la categoría moral, seguida de las categorías afectivas e individual. Reflejándose con este hecho la primacía, por parte de los futuros profesionales de la educación, del respeto por las normas morales que promueven una mejor convivencia en sociedad, el fomento del desarrollo identitario y el bienestar personal y afectivo de cada individuo. Para corroborar este hecho, podemos resaltar que los valores que han obtenido una mayor puntuación dentro de cada una de estas categorías han sido el respeto, la felicidad y el crecimiento personal, respectivamente. Por el contrario, los deberes, el matrimonio y la superación son los peor valorados en cada una de estas categorías.

En cuanto a las categorías de valores que han obtenido una menor puntuación por la muestra y se sitúan, por lo tanto, en las últimas posiciones de la jerarquía, encontramos la instrumental, la de participación política y la religiosa. Este hecho invita a reflexionar sobre la sociedad postmodernista en la que nos encontramos ya que, siguiendo a Rawls (1991) y Lipovetsky (1990), los jóvenes actuales no conciben su realidad sin consumo. Este hecho entra en contradicción con que nuestra muestra sitúe en antepenúltimo lugar a los valores instrumentales. En este mismo sentido, analizando el valor concreto de esta categoría que ha obtenido una mayor puntuación, encontramos que los jóvenes resaltan el valor ahorrar por encima del resto. Esta situación nos incita a cuestionarnos si quizás los jóvenes italianos, ante las últimas dificultades acaecidas, se plantean cambiar.

A diferencia de lo que acabamos de comentar, las dos últimas posiciones de la jerarquía si responden a los patrones actuales de la sociedad, ya que como reflejan González-Anleo (2017) y López-Ruiz (2017), los jóvenes muestran un desinterés hacia las cuestiones políticas al considerar que estas no responden a sus intereses. Si profundizamos en sus preferencias, sitúan el vocablo democracia como el más valorado de 
la categoría, mientras que rechazan el término monarquía, lo cual subraya un deseo de tener voz y poder elegir, aunque no sean muy participativos.

La categoría de valores situada en última posición es la religiosa, siendo el valor con mayor puntuación en esta categoría la libertad religiosa y el peor puntuado confesar. Este hecho refuta las investigaciones de Lipovetsky (2016) sobre el concepto de religión a la carta y la preferencia de la sociedad sobre qué creer y cómo actuar, desde un plano religioso, según su experiencia y necesidades.

Analizada la jerarquía de valores de los futuros profesionales de la educación, podemos concluir que existe la necesidad de potenciar la educación axiológica en todos los niveles educativos. Resaltando especialmente la formación universitaria de los jóvenes que desarrollarán su labor en el ámbito educativo. Para ello, es necesario crear espacios donde reflexionar e interiorizar el papel fundamental que ejercerán durante su vida profesional. Con todo ello, podremos potenciar una educación holística, que permita formar una ciudadanía reflexiva y crítica que cuente con altas competencias tanto profesionales como personales.

\section{REFERENCIAS}

Álvarez, J. (2002) Análisis descriptivo de los valores sentimiento y emoción en la formación de profesores en la Universidad de Granada. Profesorado, revista de currículum y formación del profesorado, 6 (1-2), 1-13.

Álvarez, J. (2004). El valor familia en la formación de los educadores del mañana. Revista de Ciencias de la Educación, (197), 93-111.

Álvarez, J. (2005) Análisis de los valores en los futuros profesionales de la escuela de formación del profesorado de la Universidad de Granada. REXE. Revista de Estudios y Experiencias en Educación, 4(7), 93-109

Álvarez, J. (2007). Los valores afectivos en la formación inicial del profesorado. Estudio inicial. Cuestiones Pedagógicas, (18), 121-141.

Álvarez, J., y Rodríguez, C. (2008). El valor de la institución familia en los jóvenes universitarios de Granada. Bordón, 60(1), 7-21.

Álvarez, J., Rodríguez, C., y Lorenzo, O. (2007) Investigación sobre los valores de los jóvenes universitarios españoles. Un estudio comparado. SOCIOTAM 17(1), 25-46.

Andrés, C., y Fernández, A. (2016). Las prácticas de crianza de los padres: su influencia en las nuevas problemáticas en la primera infancia. Revista nacional e internacional de educación inclusiva, 9(1), 30-42.

Angelucci, L., Da Silvia, J., Juárez, J., Serrano, A., Lezama, J., y Moreno, A. (2009). Valores y factores sociode- mográficos en estudiantes universitarios: un estudio comparativo. Acta colombiana de psicología, 12(1), 151-162.

Arango, O., Clavijo, S, Puerta, C., y Sánchez, J. (2014). Formación académica, valores, empatía y comportamientos socialmente responsables en estudiantes universitarios. Revista de la Educación Superior, 43(1), 89-105.

Bauman, Z. (2007). Los retos de la educación en la modernidad líquida. Gedisa.

Bonilla-Borrego, J., Loscertales-Abril, F., y Páez-Morales, M. (2012). Educación en valores a través del cine (un método para estudiantes de secundaria obligatoria). PíxelBit. Revista De Medios Y Educación, (41), 127-131.

Bronfenbrenner, U. (2009). The ecology of human development: Experiments by nature and design. Harvard University Press

Cámara, A.M. (2003). Los valores en la formación de maestros. Análisis de los programas de estudio de la Universidad de Jaén. Universidad de Jaén.

Casado, A., y Sánchez-Gey, J. (1999). Valores y Educación: una investigación sobre «perfiles Valorativos». Tendencias Pedagógicas, (4,) 141-155.

Casares, P. (1995). Test de valores. Un instrumento para la evaluación. Revista Española de Pedagogía, (202), 513-537. 
Cívico, A., González, E., y Colomo, E. (2019). Análisis de la percepción de valores relacionados con las TIC en adolescentes. Revista Espacios, 40(32), 18. https:// www.revistaespacios.com/a19v40n32/19403218.html

Cívico, A., Colomo, E., y González, E. (2020). Religious Values and Young People: Analysis of the Perception of Students from Secular and Religious Schools (Salesian Pedagogical Model). Religions, 11(8), 415. https:// doi.org/10.3390/rel11080415

Codina, N., y Pestana, J. V. (2008). Investigación del ocio y del self desde el paradigma de la complejidad. Una aplicación de la técnica del presupuesto de tiempo y del Twenty-Statement Test. En E. Aguilar Gutiérrez (Ed.), El influjo del tiempo en la vivencia del ocio: transformaciones, oportunidades y riesgos en la sociedad apresurada (pp. 135-147). Universidad de Deusto.

Cohen, L., Manion, L., y Morrison K. (2011). Research methods in education. Routlegde.

Colomo, E., y Aguilar, A.I. (2019). ¿Qué tipo de maestro valora la sociedad actual? Visión social de la figura docente a través de Twitter. Bordón, 71(4), 9-24. https:// doi.org/10.13042/Bordon.2019.70310

Colomo, E., Aguilar A.I., y Ruíz, J. (2020b). \#DiaMundialDelDocente: percepción social de la figura del docente en España a través de Twitter. EDMETIC, Revista de Educación Mediática y TIC, 9(2), 181-201. https://doi. org/10.21071/edmetic.v9i2.12293

Colomo, E., Gabarda, V., y Motos, P. (2018). Pedagogía de la muerte: estudio sobre la ansiedad ante la muerte en profesionales de la educación. INNOEDUCA. Internacional Journal of Technology and Educational Innovation, 4(1), 62-70. https://doi.org/10.24310/innoeduca.2018.v4i1.4129

Colomo, E., Gabarda, V., Cívico, A., y Cuevas, N. (2020a). Percepción de estudiantes sobre el uso del videoblog como recurso digital en educación superior. Pixel-Bit. Revista de Medios Y Educación, (59), 7-25. https://doi. org/10.12795/pixelbit.74358

Cruz, P., y Santiago, P. (1999). Juventud y entorno familiar. Instituto de la Juventud.
Cubo, S., Martín, B., y García, J. L. (2011). Métodos de investigación y análisis de datos en Ciencias Sociales y de la Salud. Pirámide.

Elzo, J. (1999). Los jóvenes españoles y la iglesia: una relación asimétrica. Sal terrae: Revista de teología pastoral, (1022), 289-308.

Esteban, F. (2018). Ética del profesorado. Herder.

Esteve, J. M. (2006). Las emociones en el ejercicio práctico de la docencia. Teoría de la educación, 18, 85-107.

Flammer, A., y Alsaker, F. D. (2005). Adolescents in school. En L. Goossens y S. Jackson (Eds.), Handbook of adolescent development: European perspectives (pp. 223245). Psychology Press.

Fradejas, E., y Espalda, M. (2018). Evaluación de la motivación en adolescentes que practican deporte en edad escolar. Retos. Nuevas tendencias en Educación Física, Deportes y Recreación, (33), 27-33.

García Hoz, V. (1976). Test de reacción valorativa. Bordón, (214), 245-270.

Gervilla, E. (1993). Postmodernidad y educación: valores y cultura de los jóvenes. Dykinson.

Gervilla, E. (2000a). Un modelo axiológico de educación integral, Revista Española de Pedagogía, (215), 39-58.

Gervilla, E. (2000b). Valores de la Educación Integral. Bordón, 52(4), 523-535.

Gervilla, E. (2004). Buscando valores. El análisis de contenido axiológico. Perfiles educativos, 26(103), 95-110.

Gervilla, E. y Otros (2018). Test de Valores Adaptado (TVA_ adaptado). Registro de la propiedad intelectual. No. 04/2017/1538.

Gil, F. (2001). Educación y crisis del sujeto. Teoría de la educación, 13, 45-68.

Goicoechea, M.A. (2010). Análisis de los valores de una organización de educación no formal: pioneros (Tesis doctoral). Universidad de La Rioja, La Rioja, España.

González-Anleo, J.M. (2017). Valores morales, finales y confianza en las instituciones: un desgaste que se acelera. En J.M. González-Anleo y J.A. López-Ruiz (Dirs.), 
Jóvenes españoles entre dos siglos 1984-2017 (pp. 1352). Fundación SM.

Gramigna, A., y Contreras, F.S. (2010). Etica e formazione nell'era della nanotecnologie. Pedagogika.it, 4, 79-85.

Gutiérrez, M., Tomás, J. M., y Calatayud, P. (2017). Influencia del clima motivacional en educación física sobre las metas de logro y la satisfacción con la vida de los adolescentes. Retos. Nuevas tendencias en Educación Física, Deportes y Recreación, (31), 157-163.

Hermoza, J.P (2016). Correlación del matrimonio civil y los casos de unión de hecho en el Derecho familiar. Lex, 14(17), 129-146.

Herrera-Mendoza, K., Acuña, M., Ramírez, M.J., y De La Hoz, M. (2016). Actitud y conducta pro-ecológica de jóvenes universitarios. Opción, 32(13), 456-477.

Lipovetsky, G. (1990). El imperio de lo efímero. La moda ysu destino en las sociedades modernas. Anagrama.

Lipovetsky, G. (2016). La era del vacío. Anagrama.

López-Ruiz, J.A. (2017). La centralidad de la familia para los jóvenes: convivencia, libertad y educación. En J.M. González-Anleo y J.A. López-Ruiz (Dirs.), Jóvenes españoles entre dos siglos 1984-2017 (pp. 105-164). Fundación SM.

Lüdecke-Plümer, S. (2007). La enseñanza de valores y principios morales en los centros de Formación Profesional. Revista Europea de Formación Profesional, (41), 115-128.

Maldonado, M.E., Brito, M.A., Lara, G.G., Pacciotta, E., Angulo I.M., y Barreat, Y. (2016). Autoestima y perfil motivacional en jóvenes con intenciones de practicarse cirugía estética. Multiciencias, 16(1), 69-75.

Martínez, M., Buxarrais, M.R., y Esteban, F. (2002). Ética y formación universitaria. Revista Ibero Americana de Educación, (29), 17-43.

Nava-Preciado, J. M., y Ureña-Pajarito, J. H. (2017). Dominios y valoraciones sobre la felicidad en adolescentes de Guadalajara - México. Revista Latinoamericana de Ciencias Sociales, Niñez y Juventud, 15(1), 443-454.
Poletti, G. (2019). La parola agli insegnanti. Raccogliere e analizzare dati per avere informazioni. En A. Gramigna (Ed.), Dipendenza da Internet - Stili cognitivi e nuove criticità nell-apprendimento (pp.71-106). Volta a la Carta.

Prieto, E. (2008). El papel del profesorado en la actualidad. Su función docente y social. Foro de Educación, (10), 325-345.

Quijano, D., y Lorenzo, O. (2011) Percepción sobre valores en estudiantes de la Universidad Autónoma de Yucatán. En H. Casanova (Presidencia) XI Congreso Nacional de Investigación Educativa (pp. 1-11). Consejo Mexicano de Investigación Educativa, Mexico.

Rawls, J. (1991). Una teoria della giustizia. Feltrinelli.

Río, D., García, J.L., y Gil, J.A. (2010). Métodos de Investigación en Educación. UNED.

Rodríguez, C., Herrera, L., Loreno, O., y Álvarez, J. (2008). El valor de la familia en estudiantes universitarios de España: análisis y clasificación. Enseñanza e Investigación en Psicología, 21(1), 42-49.

Rodríguez-Sabiote, C., Álvarez, J., López, J.A., y Campos M.N. (2019). Análisis y establecimiento de una jerarquía de valores del alumnado de ciclos formativos de grado medio-superior de formación profesional mediante un proceso de triangulación analítica. Revista Complutense de Educación, 30(1), 109-129. https://doi. org/10.5209/RCED.55557

Salcedo, F.E. (2012). Papel del profesor en la enseñanza de estrategias de aprendizaje.Didasc@lia: Didáctica y Educación, 3(3), 17-28.

Sánchez Rodríguez, P.A., Alfageme González, M.B., y Serrano Pastor, F.J. (2010). Aspectos sociales de los videojuegos, Revista Latinoamericana de Tecnología Educativa RELATEC, 9(1), 43-52.

Schwartz, S., y Boehnke, K. (2004). Evaluating the structure of human values with confirmatory factor analysis. Journal of Research in Personality, 38(3), 230-255. https://doi.org/10.1016/S0092-6566(03)00069-2

Van-Peer, W., Hakemulder, F., y Zyngier, S. (2012). Scientific methods for the humanities. John Benjamins. 